\title{
NEAR-INFRARED SPECTROSCOPY OF CENTAURS AND IRREGULAR SATELLITES
}

\author{
Michael E. BROWN ${ }^{1}$ \\ Division of Geological and Planetary Sciences, California Institute of Technology, Pasadena, CA 91125; mbrown@gps.caltech.edu \\ Received 1999 July 13; accepted 1999 November 5
}

\begin{abstract}
We have obtained near-infrared spectra of the four brightest known Centaurs and of five irregular satellites of giant planets. Many of these objects show 1.5 and $2.0 \mu \mathrm{m}$ absorption bands indicative of water ice, including $1997 \mathrm{CU}_{26}$, Pholus, Phoebe (S9), and Nereid (N2). The satellites of Jupiter-Elara, Himalia, and Pasaphae (J6, J7, J8) - appear spectrally featureless, consistent with asteroidal origins for these bodies. Phoebe's surface water ice indicates that it formed as an icy planetesimal rather than as an asteroid.
\end{abstract}

Key words: Kuiper belt, Oort cloud - minor planets, asteroids -

planets and satellites: individual (Elara, Himalia, Nereid, Pasaphae, Phoebe)

\section{INTRODUCTION}

The isolated small bodies of the outer solar system contain some of the least processed materials remaining from the initial protosolar nebula. Whereas asteroids have been substantially heated during and after formation, comets are heated as they approach close to the sun, and outer planet regular satellites presumably formed in higher pressure nebulae surrounding the planets, isolated bodies such as the Kuiper belt objects (KBOs) formed in the cold low-pressure background nebula, which is most representative of the overall presolar chemical and physical conditions.

Near-infrared spectroscopy offers the best method for remote determination of the composition of the volatile surface component of outer solar system bodies. Such observations have shown the presence of $\mathrm{CH}_{4}, \mathrm{CO}, \mathrm{H}_{2} \mathrm{O}$, and $\mathrm{N}_{2}$ ices on Pluto (Cruikshank et al. 1987), these ices plus $\mathrm{CO}_{2}$ on Triton (Brown et al. 1995), and water ice on Charon (Buie et al. 1987) and the moons of the outer planets. Unfortunately, most KBOs are too small and too distant to obtain adequate near-infrared spectra. To circumvent the problems associated with the dimness of the KBOs, we have obtained near-infrared spectra of Centaurs and irregular satellites of the giant planets. Centaurs are the presumed transition objects between trans-Neptunian KBOs and short-period comets (see Levison \& Duncan 1997) and are on unstable orbits between the giant planets. Irregular satellites are satellites on distant, possibly inclined and eccentric orbits around their planets. They are assumed to have been captured at some point in their history, though their initial origin is unclear.

The Centaurs and irregular satellites are at closer heliocentric distances than KBOs, so they are generally brighter and spectra can be obtained more readily. Unfortunately, their smaller heliocentric distances also imply higher temperatures, so these objects suffer processing beyond that experienced by KBOs. An example of volatile loss with heliocentric distance is show in Figure 1 where we plot an estimate of the time rate of sublimation of various ices, calculated by assuming equilibrium between the temperature-dependent vapor pressure of the ice (Lide

\footnotetext{
${ }^{1}$ Alfred P. Sloan Research Fellow.
}

1996) and the pressure of the escaping gas immediately above the surface of the object. The Centaurs and irregular satellites are unlikely to retain their most volatile ices, and thus their compositions will reflect a combination of initial conditions and reactions to heating as the objects move closer to the sun.

\section{OBSERVATIONS}

Observations of the Centaurs Chiron, Pholus, 1997 $\mathrm{CU}_{26}, 1995 \mathrm{GO}$, and the irregular satellites Himalia (J6), Elara (J7), Pasaphae (J8), Phoebe (S9), and Nereid (N2) were obtained on four nights between 1998 March 7 and August 3 using NIRC, the facility near-infrared camera at the Keck telescope (Matthews \& Soifer 1994). A journal of the observations is given in Table 1 . We identified each object by its motion with respect to background stars in direct $K$-band images. With the telescope guiding at the predicted rate of the object, we then placed the object into the spectral slit and inserted the 120 line $\mathrm{mm}^{-1}$ grism and an $H$ - through $K$-band order sorting filter into the light path, allowing us to collect a spectrum in first order from 1.4 to $2.5 \mu \mathrm{m}$ at a resolution of approximately $\lambda / \Delta \lambda=100$.

Total integration time varied for each object, but the observing procedure was identical. The spectra were obtained by positioning the object in the center of the long slit, integrating for 100,200 , or $300 \mathrm{~s}$, and then offsetting the telescope $5^{\prime \prime}$ and $10^{\prime \prime}$ north and then south and obtaining similar exposures. For Pholus, Himalia, and Nereid, this procedure was repeated. For each target a calibration spectrum of a nearby star at a similar air mass was also obtained.

Data reduction was performed identically for each target. First, a flat-field image was created by median-combining multiple spectra of a diffusely illuminated dome spot and subtracting a dark image obtained with identical integration times and the cold stop inserted in the filter wheel. Next we removed the curvature of the skylines with respect to the detector columns by creating summed spectra every 16 rows, performing cross-correlations between the spectra to measure the curvature, and shifting the two-dimensional spectrum row-by-row to straighten the skylines. Typical shifts at the extreme ends of the detector were usually one or two pixels. We then differenced adjacent pairs of spectra to remove the majority of the sky background, and we divided by the flat field. Residual sky background in the subtracted 
TABLE 1

JOURNAL OF OBSERVATIONS

\begin{tabular}{|c|c|c|c|c|c|c|}
\hline Object & $\begin{array}{l}\text { Date }^{\mathrm{a}} \\
\text { (UT) }\end{array}$ & $\begin{array}{l}\text { Integration Time } \\
\text { (s) }\end{array}$ & Air Mass & $\begin{array}{l}\text { Slit Width } \\
\text { (arcsec) }\end{array}$ & $\begin{array}{c}\text { Calibrator } \\
\text { (SAO/Spectral Type) }\end{array}$ & Air Mass \\
\hline Chiron & 03 Aug 0720 & 500 & $1.76-1.83$ & 0.52 & $159027 / \mathrm{A} 0$ & 1.72 \\
\hline Pholus ............ & 08 Mar 1040 & 2000 & $1.22-1.05$ & 0.52 & 100912/A3 & $1.16-1.08$ \\
\hline $1997 \mathrm{CU}_{26} \ldots \ldots \ldots$ & 07 Mar 0855 & 1000 & $1.03-1.05$ & 0.52 & 098558/A2 & 1.10 \\
\hline 1995 GO .......... & 07 Mar 1324 & 1000 & $1.33-1.34$ & 0.52 & $182160 / \mathrm{A} 2$ & 1.41 \\
\hline Himalia (J6) ........ & 02 Aug 1114 & 2000 & $1.32-1.20$ & 0.52 & 147039/A0 & 1.18 \\
\hline Elara $(\mathrm{J} 7) \ldots \ldots \ldots$ & 02 Aug 1213 & 1500 & $1.14-1.11$ & 0.52 & 147039/A0 & 1.09 \\
\hline Pasaphae (J8)...... & 02 Aug 1308 & 1500 & $1.09-1.08$ & 0.52 & 147039/A0 & 1.09 \\
\hline Phoebe (S9)........ & 02 Aug 1355 & 1500 & $1.13-1.09$ & 0.52 & $147039 / \mathrm{A} 0$ & 1.09 \\
\hline Nereid (N2)....... & 15 Jun 0342 & 3000 & $1.32-1.52$ & 0.37 & 032071/G5 & 1.45 \\
\hline
\end{tabular}

${ }^{a}$ All dates 1998.

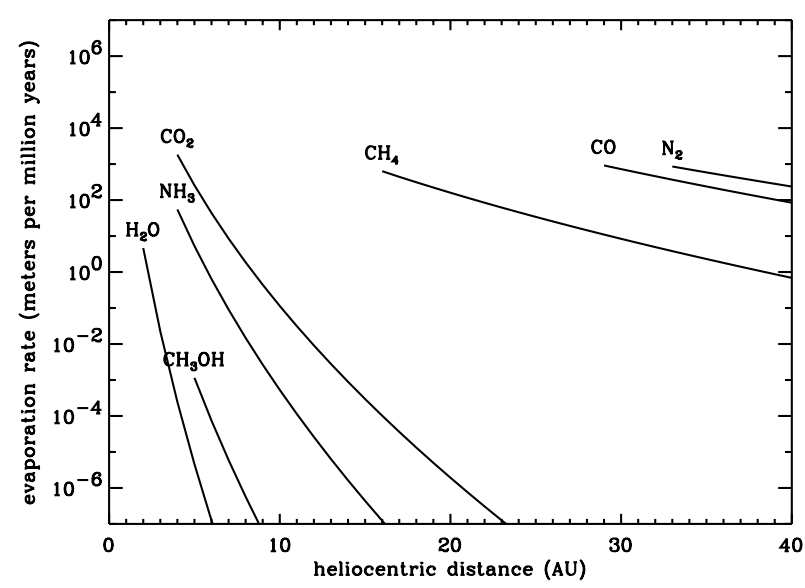

FIG. 1.-Sublimation rate of pure ices as a function of heliocentric distance. The curves are truncated on the low heliocentric end when the temperature reaches the melting point of the ice. The curve for $\mathrm{CH}_{3} \mathrm{OH}$ is calculated by extrapolating from the vapor pressure of liquid methanol and is therefore slightly higher than the true evaporation rate of solid methanol ice.

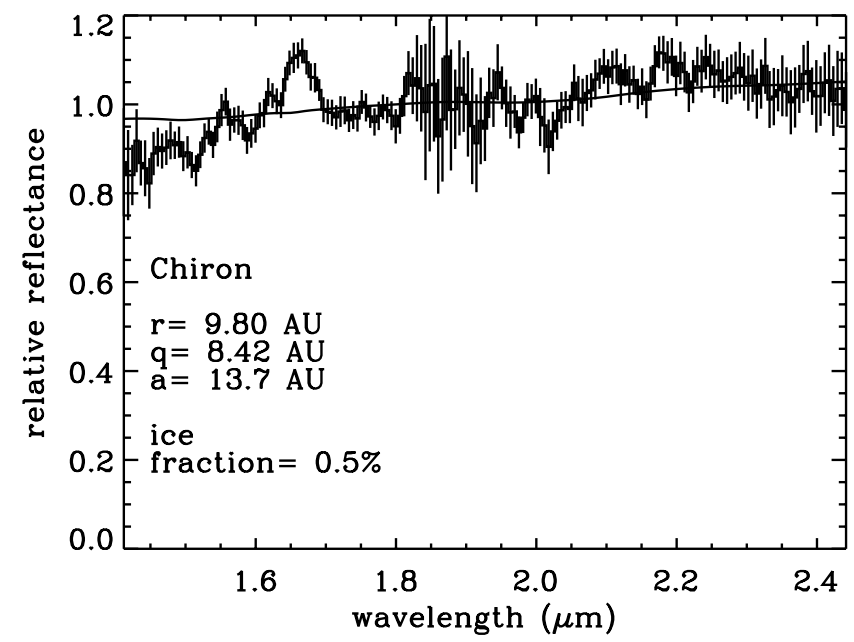

FIG. 2.- Near-infrared spectrum and model of Chiron scaled to a value of 1.0 at $1.8 \mu \mathrm{m}$. Error bars show the uncertainty in the sky subtraction at each wavelength. Systematic errors dominate over these random errors (see text for details). The parameters $r, q$, and $a$ are the heliocentric distance, the perihelion distance, and the semimajor axis of the object. The ice fraction is the best-fit areal coverage of water ice.

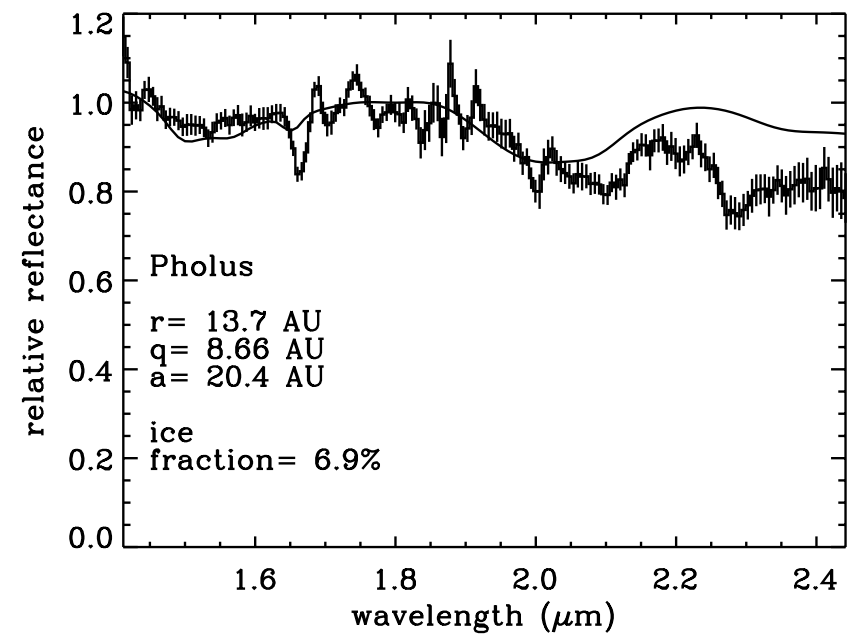

FIG. 3.-Near-infrared spectrum and model of Pholus. The non-waterice absorption features have been attributed to methanol or other light hydrocarbons by Cruikshank et al. (1998).

image (resulting from changing sky background between two adjacent exposures) was removed by measuring the residual sky spectrum in 20 pixel-wide swaths above and below the target and subtracting the average of these spectra from every row of the spectrum. The object spec-

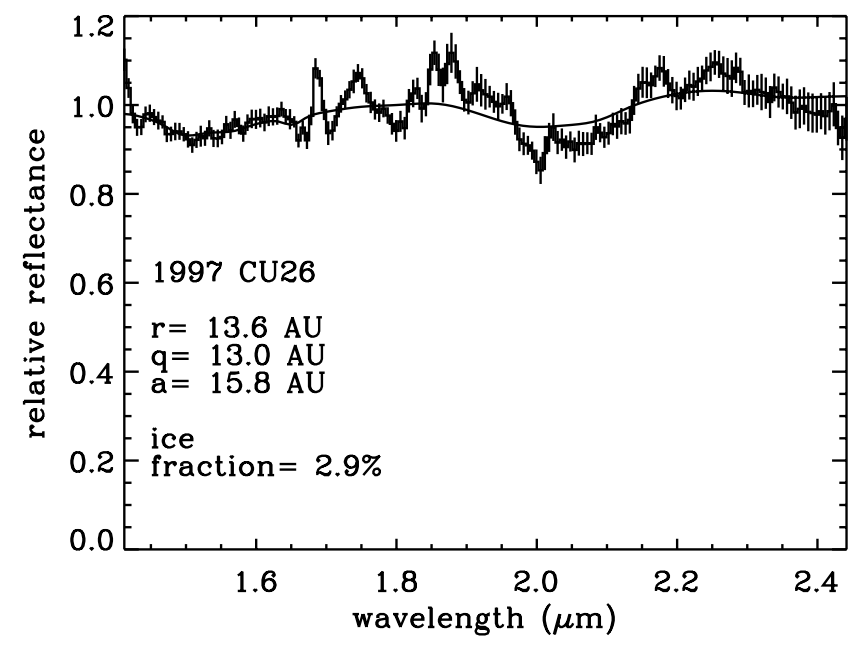

FIG. 4.- Near-infrared spectrum and model of $1997 \mathrm{CU}_{26}$ 


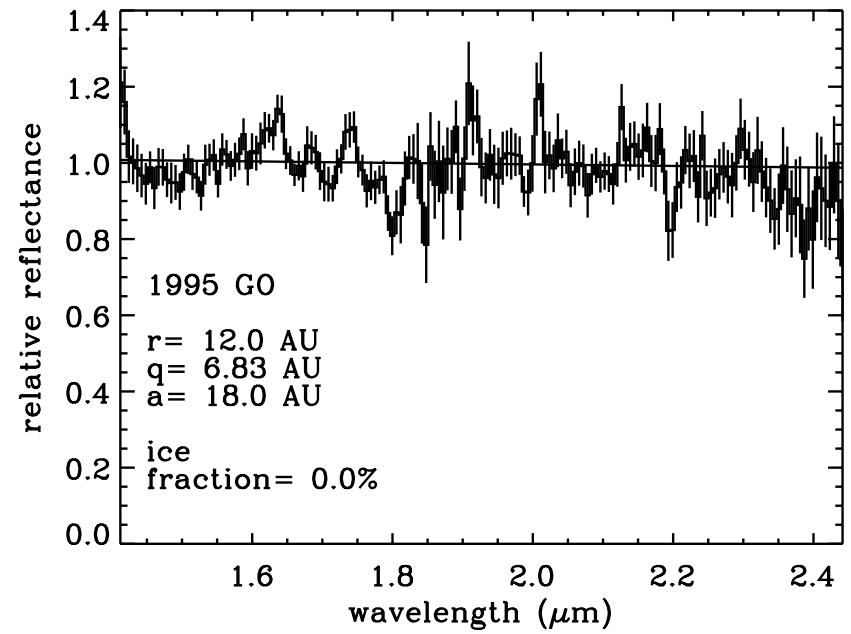

FIG. 5.-Near-infrared spectrum and model of 1995 GO. An upper limit of $\sim 1 \%$ ice fraction could be fitted within the noise of the data.

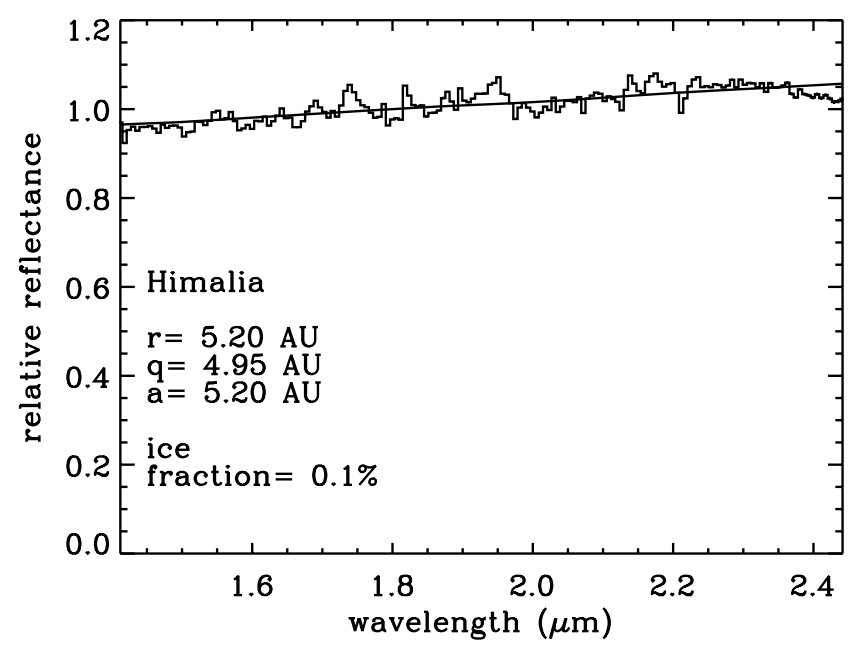

FIG. 6.-Near-infrared spectrum and model of Himalia (J6). The bestfit ice fraction of $0.1 \%$ is consistent with zero. This spectrum is consistent with an asteroidal origin for the Jovian irregular satellites.

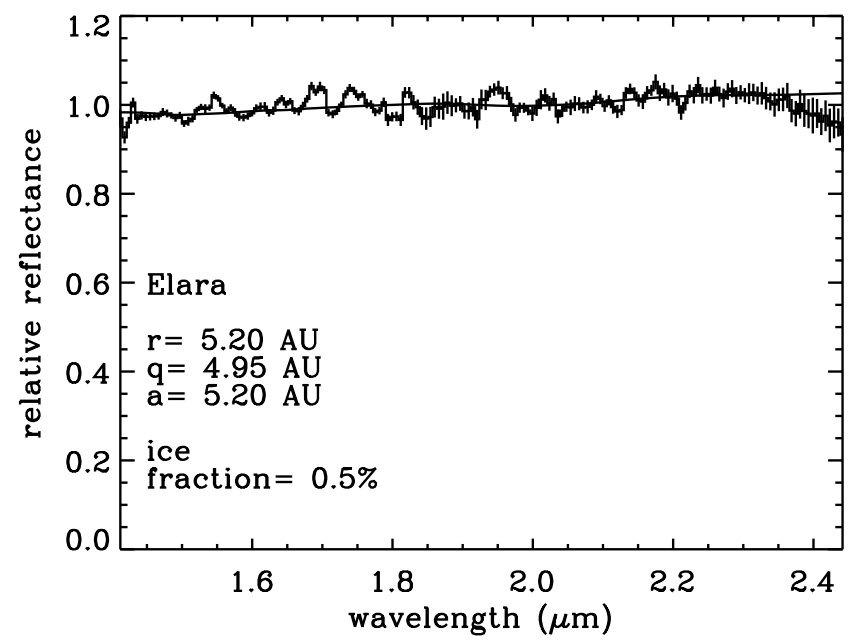

FIG. 7.- Near-infrared spectrum and model of Elara (J7). The best-fit ice fraction of $0.5 \%$ is consistent with zero.

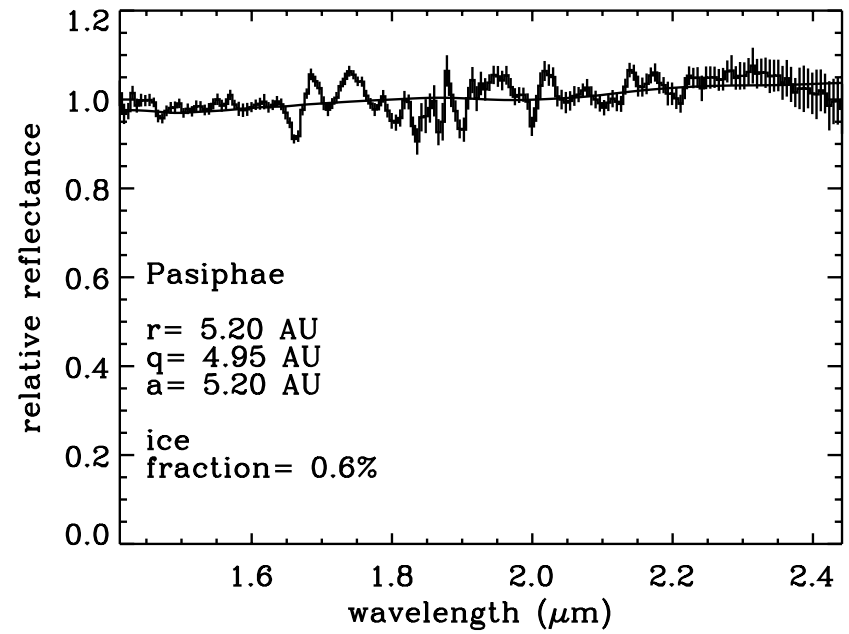

FIG. 8.-Near-infrared spectrum and model of Pasaphae (J8). The bestfit ice fraction of $0.6 \%$ is consistent with zero.

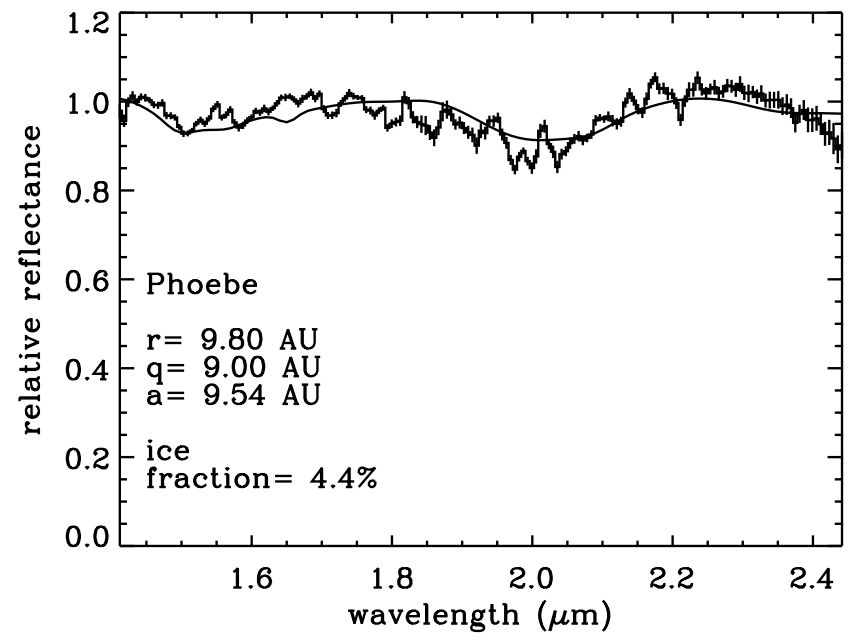

FIG. 9.- Near-infrared spectrum and model of Phoebe (S9). The presence of water ice on the surface of Phoebe demonstrates that it is a captured outer solar system body, rather than an asteroid.

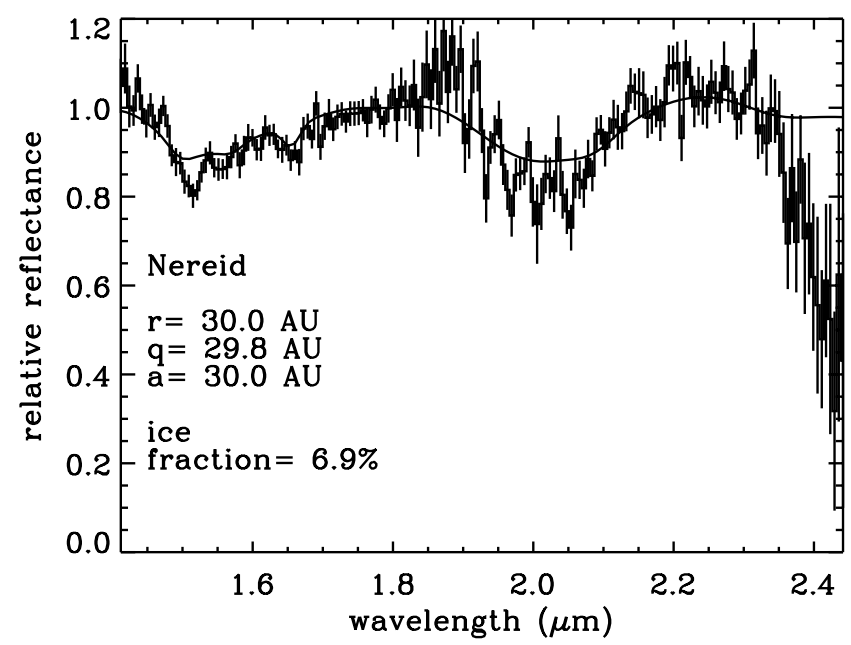

FIG. 10.-Near-infrared spectrum and model of Nereid (N2). This spectrum shows the clearest evidence of the $1.65 \mu \mathrm{m}$ absorption present in crystalline water ice but absent in amorphous ice. 
trum was extracted by summing 10 rows weighted by the signal at each spatial position. Individual spectra were examined by eye to remove bad pixels and all spectra of a target object were summed to create the total spectrum.

Calibrator stars were reduced identically except that exposures were so short that skylines were not visible, so the spectral curvature was removed by using the curvature from the most recently measured target star. The object spectrum was then divided by the calibrator star spectrum to remove the effects of telluric absorption. Most calibrator stars were of early-A spectral type, which are known to have hydrogen Brackett absorption lines, with the strongest at $1.61,1.64,1.68,1.74,1.82,1.95$, and $2.16 \mu \mathrm{m}$ (Lançon \& Rocca-Volmerange 1992). No apparent emission-feature artifacts in the divided spectra are visible at these wavelength. The wavelength scale was set by matching to the known wavelengths of $\mathrm{OH}$ emission features in the night sky. Finally, we converted this divided spectrum to a relative spectral reflectance by multiplying by a blackbody function at the temperature of the calibrator star and dividing by a blackbody function at the $5770 \mathrm{~K}$ temperature of the sun. The final spectrum shows the relative reflectance of the object when viewed with white light. The extracted spectra are shown in Figures 2-10. Error bars show the uncertainty in the sky subtraction at each wavelength. At most wavelengths, the systematic errors due to slight wavelength shifts and sky transparency changes between the target and calibrator star dominate over the random sky subtraction errors.

\section{SPECTRAL MODELING}

Many of the spectra of the Centaurs and irregular satellites show the 1.5 and $2.0 \mu \mathrm{m}$ absorption features characteristic of water ice. To quantify relative amounts of water ice present, we attempt to model each spectrum as a simple areal mixture of water ice and a dark spectrally featureless component. As not all of our objects have measured albedos, we simply model the relative reflectance in all cases and scale to a model value of 1.0 and $1.8 \mu \mathrm{m}$. For water ice, we calculate the spectrum from the laboratory optical constants of Grundy \& Schmitt (1998) assuming grain sizes of $10 \mu \mathrm{m}$ and temperatures appropriate for the blackbody temperature at the objects' distance. Hapke theory (Hapke 1991) is used to transform the optical constants to a reflectance spectrum. The dark component we model as a featureless but possibly slightly red or blue continuum. For each model spectrum, we vary the fractional coverage of water ice and the slope of the dark continuum to minimize the residuals with the object spectrum. The numeric best-fit value of fractional ice coverage is only meant to be taken as an indication of the depth of the water absorption features; the models are no means unique and many different combinations of water-ice grain sizes and mixing spatial scales can be used to fit weak water-ice absorption features (Davies et al. 1997). The best-fit two component models are shown in Figures 2-10. Individual spectra and models are discussed below.

\section{DISCUSSION}

\subsection{Centaurs}

Chiron. The spectrum of Chiron shows only weak evidence for the presence of water ice (Fig. 2); we regard the best-fit value of $0.5 \%$ surface coverage as, at best, an upper limit to the presence of these absorption features. Previous spectroscopy by Luu, Jewitt, \& Cloutis (1994) also showed Chiron to be spectrally flat, which they suggested could be the result of activity on Chiron burying the icy surface. Recently, however, Foster et al. (2000) have shown convincing evidence for a weak absorption at $2.0 \mu \mathrm{m}$ with a depth of approximately $5 \%$, which they attribute to water ice. Their spectrum covers only the 1.6 to $2.2 \mu \mathrm{m}$ range, so they cannot discern the $1.5 \mu \mathrm{m}$ band. The $2.0 \mu \mathrm{m}$ absorption is consistent with the depression that is seen in our spectrum at $2.0 \mu \mathrm{m}$ (indeed, if we include only the 1.6 to $2.2 \mu \mathrm{m}$ data, our best model fit gives $4 \%$ water ice coverage), but our spectrum, which reaches to $1.4 \mu \mathrm{m}$, shows no evidence of the $1.5 \mu \mathrm{m}$ absorption that should be present for water ice. Instead, a general downward trend is seen below $1.6 \mu \mathrm{m}$, which could possibly be due to both water ice and an additional absorber. All other spectra showing water ice, however, distinctly show the upturn between 1.5 and 1.4 $\mu \mathrm{m}$.

The status and abundance of water ice on Chiron is uncertain, but regardless of the precise amount, the water ice spectral features on the surface of this Centaur are clearly smaller than those of the Centaurs Pholus (Fig. 3) and $1997 \mathrm{CU}_{26}$ (Fig 4.), and the irregular satellite Phoebe (Fig. 9). Chiron approaches closer to the sun than do these other objects, so the additional heating could cause the observed spectral characteristics. However, water ice should not begin to sublime strongly until the object is well into the inner solar system (Fig. 1). Nonetheless, Chiron had been observed to be sporadically active much further from the sun (Tholen, Hartmann, \& Cruikshank 1988; Luu \& Jewitt 1990), which is likely to have some effect on the optical properties of its surface, though precisely what effect is not clear. It is thus tempting to attempt to explain this diminished water ice signature as a consequence of activity in Chiron, as the other objects with ice signatures have had no activity detected. However, no convincing cause of this diminishing surface water ice has yet been proposed.

Pholus. The spectrum of Pholus is unique in that it is the only of the Centaurs to show clear evidence of an absorption feature other than those of water ice. Recent modeling by Cruikshank et al. (1998) suggests that this feature could be due to methanol ice on the surface of Pholus. Methanol absorbs at $2.1 \mu \mathrm{m}$, where our water-ice fit is bad, and more strongly at $2.3 \mu \mathrm{m}$, where the nonwater absorption appears. As pointed out by Cruikshank et al., however, the $2.3 \mu \mathrm{m}$ feature of pure methanol should actually be a doublet at 2.27 and $2.33 \mu \mathrm{m}$, which does not appear in our data. More complicated hydrocarbons will have features in roughly the same places and a better fit is likely possible; however, most potential hydrocarbon ices have not had their near-infrared spectral constants measured, so no definitive identification is possible. Methanol is abundant in interstellar medium and cometary ices (van Dishoeck et al. 1993; BockeleeMorvan et al. 1990), so it is a likely component of an outer solar system object. Methanol is only slightly more volatile than water ice (Fig. 1); heavier hydrocarbons are even less volatile. Such compounds are therefore able to survive at the elevated temperatures experienced by Centaurs.

$1997 \mathrm{CU}_{26}$. The spectrum of $1997 \mathrm{CU}_{26}$ shows the clearest evidence of water-ice absorption of any of the Centaurs. The spectrum of this object was modeled by Brown \& Koresko (1998) as a mixture of distinct surface areas of a dark neutral substance and 3\% water ice. Brown et al. (1998) obtained an equally good model fit assuming an inti- 
mate mixture of a dark neutral substance with $67 \%$ water ice. This large difference in inferred surface water ice abundance is indicative of the enormous uncertainty in trying to derive quantitative abundances from these types of spectra. $1997 \mathrm{CU}_{26}$ is currently at a similar heliocentric distance as Pholus and is only slightly larger in diameter than Pholus (Jewitt \& Kalas 1998), so a similarity between these objects might be expected. The water-ice absorption bands on 1997 $\mathrm{CU}_{26}$ are similar in depth as those as Pholus, but 1997 $\mathrm{CU}_{26}$ shows no hint of the $2.3 \mu \mathrm{m}$ absorption attributed to methanol on Pholus. In addition, the visible spectrum of $1997 \mathrm{CU}_{26}$ is approximately neutral, while that of Pholus is extremely red (Barucci, Lazzarin, \& Tozzi 1999). Clearly, the two objects' surface compositions are substantially different. The difference could be due to the objects' differing perihelion distances (13.1 AU for $1997 \mathrm{CU}_{26}$ vs. 8.4 for Pholus), but this possibility seems unlikely, as methanol should not sublimate heavily at the distance of either Pholus or $1997 \mathrm{CU}_{26}$. The reason for a lack of methanol on $1997 \mathrm{CU}_{26}$ or any of the other objects remains unclear.

1995 GO. The spectrum of $1995 \mathrm{GO}$ appears featureless. This was the faintest Centaur we observed, so the spectrum has lower signal to noise than the others. We find that water-ice features equivalent to $1 \%$ surface coverage would be undetectable in our spectrum, but those as strong as the ones on $1997 \mathrm{CU}_{26}$ or Pholus would be clearly visible. Dynamically, 1995 GO is similar to Chiron, in that it has a close perihelion passage (6.83 AU). Like Chiron, $1995 \mathrm{GO}$ is likely to be active during perihelion passages, so if activity on Chiron is responsible for its spectral characteristics, we might expect similar characteristics on $1995 \mathrm{GO}$; visible spectroscopy has shown that both of these objects are relatively neutrally colored, with 1995 GO slightly more red (Barucci et al. 1999). 1995 GO will go through perihelion next in 2002 and should be monitored closely for signs of activity beginning immediately.

\subsection{Irregular Satellites}

Himalia, Elara, Pasaphae (J6, J7, J8). The irregular satellites of Jupiter are thought to be captured bodies. Pollack, Burns, \& Tauber (1979) suggest that the capture occurred via gas drag in the proto-Jovian nebula and that the sudden slowing of the parent bodies caused fragmentation, leading to groups of satellites. Two separate events led separately to the prograde and retrograde groups of satellites. Alternatively, capture could have occurred when two objects collided and shattered within Jupiter's sphere of influence, leading to the prograde and retrograde groups (Colombo \& Franklin 1971). Two of the observed spectra are from the prograde group (J6 and J7) while the other is from the retrograde group (J8).

The spectra of these objects show no hints of a water-ice absorption feature or of any other feature. The spectra appear similar to those of $\mathrm{P}$ - and D-class outer asteroid belt and Trojan asteroids (Luu et al. 1994; Dumas, Owen, \& Barucci 1998). Radiometry of these objects has also shown that their albedos $\left(a_{v} \sim 3 \%\right)$ are similar to those of these types of asteroids (Cruikshank 1977), while the visible spectra appear similar to C-class asteroids (Tholen \& Zellner 1984). All available evidence continues to suggest that these satellites originated as typical outer asteroids before collision and capture in the Jovian system.

Phoebe (S9). Phoebe-unlike any of Saturn's other satellites - moves in a retrograde orbit, one which, more- over, is distant and highly inclined. These unusual orbital characteristics have led to a century of speculation that Phoebe is an asteroid gravitationally captured into the Saturnian system (Pickering 1899). By the late-1970s, telescopes had become powerful enough to discern thatunlike the regular satellites of Saturn, which were bright and found to be covered in water ice-Phoebe appeared dark, asteroid-like, and water-ice free (Degewij, Cruikshank, \& Hartmann 1980). Further observations showed that Phoebe's visible spectrum is consistent with those of the C-class asteroids that populate the middle of the asteroid belt (Tholen \& Zellner 1983), and a theory to explain how asteroids could reach the orbit of Saturn was proposed (Hartmann 1987). Low-resolution images from Voyager provided confirmation that Phoebe has a low C-class asteroid-like albedo of approximately 6\% (Thomas et al. 1983).

The near-infrared spectrum of Phoebe, rather than looking asteroid-like, however, is similar to that of 1997 $\mathrm{CU}_{26}$, showing clear indications of water ice. The albedo and visible spectrum of Phoebe are also similar to those of $1997 \mathrm{CU}_{26}$. That Phoebe resembles a Centaur rather than an asteroid should come as no surprise; the frequent passage of Centaurs past Saturn should have provided many opportunities for capture. Permanent capture of such a body, however, requires some sort of energy dissipation. Two possible forms of dissipation for the capture of Phoebe include a collision with an existing satellite and drag from the primordial gaseous envelope of the still-accreting Saturn. While Jupiter's irregular satellites come in two distinct clusters, immediately suggesting a collisional origin, the apparent lack of other irregular satellites of Saturn suggests that gas drag may have been the more likely mechanism. If so, Phoebe must have been captured early in the history of the solar system. During this epoch, the outer solar system was likely filled with icy planetesimals that had not become consolidated into giant planets. Many of these were scattered into the Oort cloud and are seen today as long period comets. In the gas-drag hypothesis, Phoebe is likely to have been one of these more locally conceived bodies, rather than something that formed beyond Neptune and scattered inward. Regardless of Phoebe's precise origin, its similarity to $1997 \mathrm{CU}_{26}$ demonstrates that Phoebe formed as an icy outer solar system planetesimal rather than a rocky asteroidal body. A similar conclusion was recently reached by Owen et al. (1999).

Nereid (N2). The satellite Nereid orbits Neptune in a highly inclined, eccentric, and distant orbit, suggesting that, like other irregular satellites in the solar system, it is possibly a captured body. Goldreich et al. (1989), however, have suggested that Nereid could have formed as a regular satellite of Neptune, but then the capture of Triton and its subsequent orbital circularization could have moved Nereid from an originally regular orbit to its current irregular orbit.

Our observations show that Nereid has the largest fraction of water ice of any of the objects observed, with waterice absorption depths comparable to some of the darker Uranian satellites. Nereid has, in addition, the largest optical albedo of the objects with known albedo, again similar to the darker Uranian satellites (see below). Brown, Koresko, \& Blake (1998) suggest that this spectral and albedo similarity between the regular Uranian satellites and Nereid is evidence that Nereid did indeed form as a regular 
satellite around Neptune. While the spectral similarity between Nereid and the Uranian satellites is striking (see Fig. 11), without a full understanding of the causes of surface compositional diversity in the outer solar system, it remains difficult to directly link surface composition to formation location.

\subsection{Comparisons}

Albedo. All of our observed objects except for 1995 GO have had radiometric or direct measurements of their visual albedos. We calculate their geometric albedo spectra by using the measured visual albedo and scaling the $2.0-2.4 \mu \mathrm{m}$ spectrum to the measured $V-K$ color (Table 2). Results for all observations are shown in Fig. 11, along with the two darkest major satellites of Uranus (From Brown 1983).

The visual albedos and water-ice absorption depths of the Uranian satellites (and Nereid) are directly related; objects with higher albedo have deeper water absorptions. The spectra can all be modeled with a single dark absorber and a varying amount of surface ice. In contrast, the remaining objects shown no albedo-water ice relationship. Phoebe, for example, shows water-ice absorption twice the

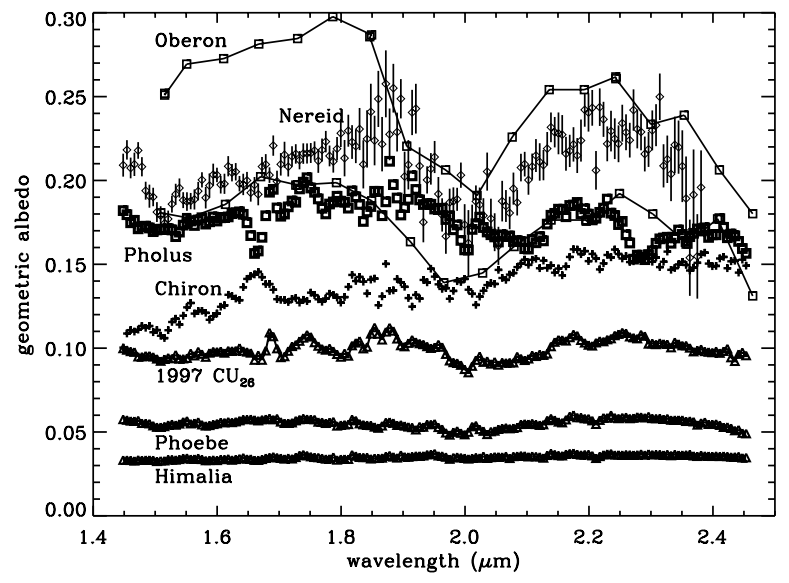

FIG. 11.-Near-infrared spectral geometric albedos for Centaurs and irregular satellites with known visible albedos. The Uranian satellites Oberon (labeled solid line) and Umbriel (unlabeled solid line) are shown for comparison.

TABLE 2

Properties of Distant Satellites and Minor Planets

\begin{tabular}{|c|c|c|c|c|}
\hline Object & $V$ Albedo & $V-K$ & $K$ Albedo & Reference \\
\hline Oberon & 0.29 & 1.2 & 0.24 & 1,2 \\
\hline Umbriel ......... & 0.20 & $1.2^{\mathrm{a}}$ & 0.17 & 1 \\
\hline Nereid............ & $0.18 \pm 0.02$ & $1.6^{\mathrm{b}}$ & 0.21 & 3 \\
\hline Chiron ........... & $0.14_{-3}^{+6}$ & 1.5 & 0.15 & 4,5 \\
\hline Pholus .......... & $0.044 \pm 0.013$ & 2.9 & 0.17 & 6,7 \\
\hline $1997 \mathrm{CU}_{26} \ldots \ldots$ & $0.045 \pm 0.01$ & 2.2 & 0.09 & 8,9 \\
\hline Himalia .......... & 0.03 & 1.7 & 0.04 & 10,11 \\
\hline Phoebe ........... & $0.069 \pm 0.02$ & 1.4 & 0.07 & 12,13 \\
\hline Sun ................ & $\ldots$ & 1.4 & $\ldots$ & 14 \\
\hline
\end{tabular}

${ }^{\text {a }}$ Assumed to be the same as Oberon.

${ }^{b}$ Measured from our spectral acquisition images.

REFERENCES.- (1) Veverka, Brown, \& Bell 1991; (2) Cruikshank et al. 1977; (3) Thomas, Helfenstein, \& Veverka 1991; (4) Campins et al. 1994; (5) Hartman et al. 1990; (6) Davies et al. 1993a; (7) Davies, Sykes, \& Cruikshank1993b; (8) Jewitt \& Kalas 1998; (9) Davies et al. 1998; (10) Cruikshank 1977; (11) Skyes et al. 2000; (12) Thomas et al. 1983; (13) Degewij et al. 1980; (13) Hardorp 1980 depth of $1997 \mathrm{CU}_{26}$, but has an infrared albedo only half as high. Chiron, with little or no water-ice absorption, has an albedo higher than either of these. Clearly, different surface compositions are responsible for the neutral dark component on each of these objects.

Ice abundance. The Centaurs and irregular satellites show no apparent spectral regularity with heliocentric distance, in either depth of water-ice absorption or continuum slope (Fig. 12). A similar lack of correlation was found by Barucci et al. (1999) in the slopes of the visible spectra of Centaurs. These objects might be more reasonably expected to show a correlation with perihelion distance, as this distance corresponds to the maximum heating the object receives. A slight correlation appears when the spectra are viewed versus perihelion distance (Fig. 13); objects that approach within about 8.5 AU of the sun show little or no sign of water ice,

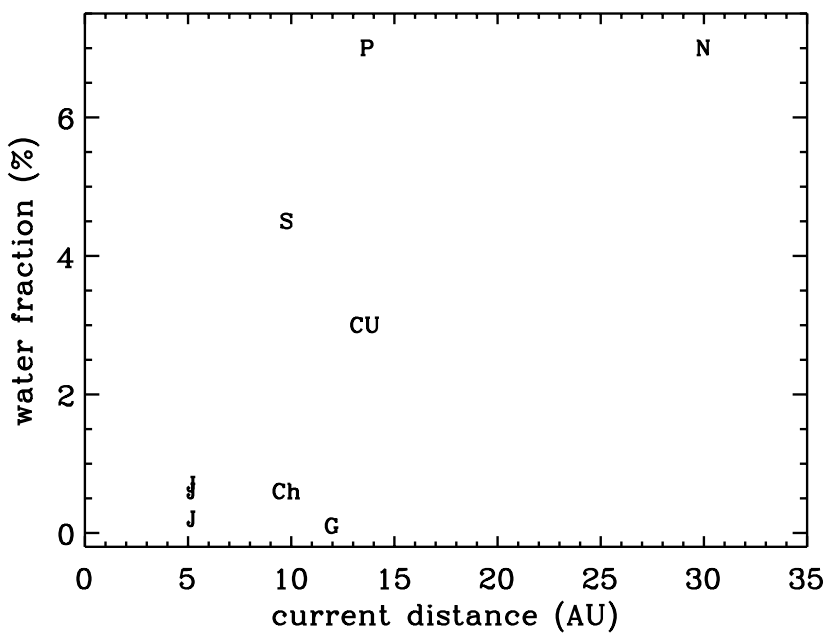

FIG. 12. - Comparison of the best-fit fractional water ice coverage as a function of heliocentric distance of the object. The labels are as follows: $\mathrm{Ch}=$ Chiron, $\mathrm{P}=$ Pholus, $\mathrm{CU}=1997 \mathrm{CU}_{26}, \mathrm{G}=1995 \mathrm{GO}, \mathrm{J}=$ Jovian irregular satellites, $\mathrm{S}=$ Saturnian irregular satellite (Phoebe), and $\mathrm{N}=$ Neptunian irregular satellite (Nereid). No consistent pattern with heliocentric distance appears.

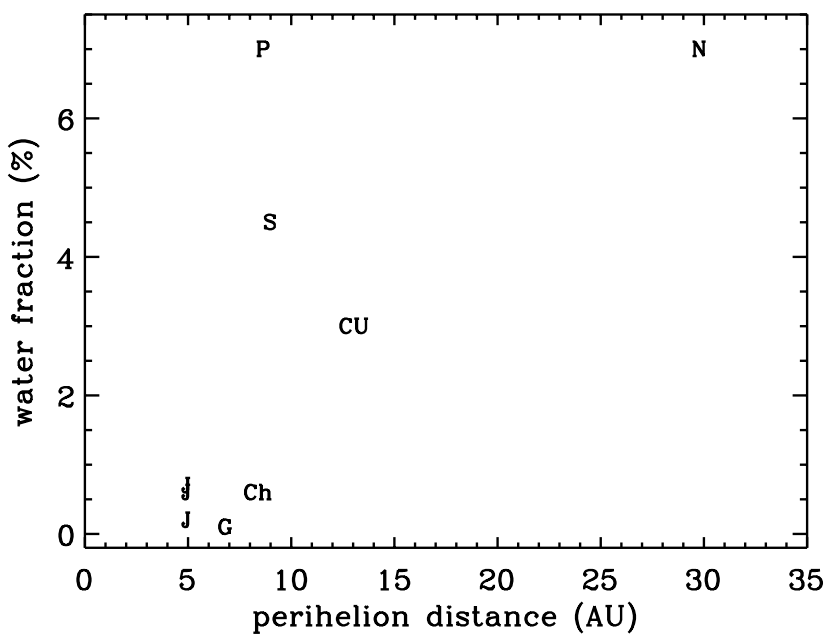

Fig. 13.-Comparison of the best-fit fractional water ice coverage as a function of perihelion distance of the object. Labels are as in Fig. 12. The perihelion distance controls the maximum heating rate the object experiences, so a correlation with this parameter is most expected. All objects with perihelion distance less than $8.5 \mathrm{AU}$ have little to no surface water ice, while all of those outside $8.5 \mathrm{AU}$ show clear signatures of surface water ice. 
while those outside this distance all show more abundant water ice. If we discount the Jovian satellites, however, which we have argued are asteroidal in origin, and Nereid, which we have argued is a regular satellite, this apparent correlation is driven by only a small number of objects, and the perihelion distance between Chiron $(q=8.42 \mathrm{AU})$, which shows little or no water-ice absorption and Pholus ( $q=8.66 \mathrm{AU})$, which shows the largest amount of water-ice absorption of any of the observed Centaurs, is small. This potential transition distance corresponds well to the distance at which sublimation of $\mathrm{CO}_{2}$ should begin to lead to significant cometary activity, such as seen in Chiron (Fig. 1). More volatile ices such as $\mathrm{CO}$ have also been observed to be important drivers of cometary activity at distances just beyond Jupiter (Senay \& Jewitt 1994; Biver et al. 1996).

Organics. Spectral evidence for the presence of organic materials is seen in the extremely red slope of the visible spectrum of Pholus and in the $2.3 \mu \mathrm{m}$ methanol-like absorption feature of this object (Cruikshank et al. 1998). The presence of such materials is not a surprise; methanol is an abundant ice in the interstellar medium and comets (van Dishoeck et al. 1993; Bockelee-Morvan et al. 1990), and it should be incorporated in outer solar system objects. Its relatively low volatility should allow it to remain present in almost all of the objects we observed. The surprise instead is that it is clearly not abundant on the surface of any of the objects other than Pholus. While it is possible that sporadic activity such as observed on Chiron could disrupt or overturn a radiation processed mantle responsible for the dark red optical spectrum, it is difficult to imagine how such activity could mask a methanol surface-ice feature if meth- anol were present throughout the object. An interesting clue to the nature of the red surface and the presence of methanol and any possible correlation would be to obtain nearinfrared spectra of more extremely red objects, such as the Centaur Nessus (Barucci et al. 1998); however, all currently known such objects are extremely faint and current spectroscopy will be difficult.

\section{SUMMARY}

Near-infrared spectroscopy of Centaurs and outer solar system irregular satellites has revealed a variety of surface compositions. Many of the objects show weak 1.5 and 2.0 $\mu \mathrm{m}$ absorption features of water ice on their surface. This water ice is mixed with or covers a dark and near-infrared neutral material, which appears to vary from object to object. An additional absorption due to methanol ice or its related products is found only on Pholus, though methanol ice should be stable on all of the objects observed. No compelling reason for the spectral differences can be discerned.

We would like to thank Chris Koresko and Geoff Blake for their generous help in obtaining these observations and an anonymous referee for useful suggestions. This research is supported entirely through private funding. These data were obtained at the W. M. Keck Observatory, which is operated as a scientific partnership among the California Institute of Technology, the University of California, and the National Aeronautics and Space Administration. The observatory was made possible by the financial support of the W. M. Keck Foundation.
Barucci, M. A., Lazzarin, M., \& Tozzi, G. P. 1999, AJ, 117, 1929

Biver, N., et al. 1996, Nature, 380, 137

Bockelee-Morvan, D., Crovisier, J., Colom, P., Despois, D., \& Paubert, G. 1990, in Proc. of the 24th ESLAB Symp., Formation of Stars and Planets and the Evolution of the Solar System, ed., B. Battrick (Paris: ESA), 143

Brown, M. E., \& Koresko, C. D. 1998, ApJ, 505, L65

Brown, M. E., Koresko, C. D., \& Blake, G. A. 1998, ApJ, 508, L175

Brown, R. H. 1983, Icarus, 56, 414

Brown, R. H., Cruikshank, D. P., Pendleton, Y., \& Veeder, G. J. 1998, Science, 280, 1430

Brown, R. H., Cruikshank, D. P., Veverka, J., Helfenstein, P., \& Eluszkiewicz, J. 1995, Neptune and Triton, ed. Cruikshank, D. P. (Tucson: Univ. Arizona Press), 991

Buie, M. W., Cruikshank, D. P., Lebovsky, L. A., \& Tedesco, E. F. 1987, Nature, 329, 522

Campins, H., Telesco, C. M., Osip, D. J., \& Rieke, G. H., Rieke, M. J., \& Schulz, B. 1994, AJ, 108, 2318

Colombo, G., \& Franklin, F. A. 1971, Icarus, 15, 186

Cruikshank, D. P. 1977, Icarus, 30, 224

Cruikshank, D. P., et al. 1998, Icarus, 135, 389

Cruikshank, D. P., Roush, T. L., Moore, J. M., Sykes, M., Owen, T. C. Bartholomew, M. J., Brown, R. H., \& Tryka, K. A. 1987, in Pluto and Charon, ed. S. A. Stern, \& D. J. Tholen (Tucson: Univ. Arizona Press), 221

Davies, J. K., Roush, T. L., Cruikshank, D. P., Bartholomew, M. J., Geballe, T. R., Owen, T., \& de Bergh, C. 1997, Icarus, 127, 238

Davies, J. K., McBride, N., Ellison, S. L., Green, S. F., \& Ballantyne, D. R. 1998, Icarus, 132, 213

Davies, J. K., Spencer, J., Sykes, M., Tholen, D., \& Green, S. 1993a, IAU Circ. 5698

Davies, J. K., Sykes, M. V., \& Cruikshank, D. P. 1993b, Icarus, 102, 166

Degewij, J., Cruikshank, D. P., \& Hartmann, W. K. 1980, Icarus, 44, 1980

Dumas, C., Owen, T., \& Barucci, M. A. 1998, Icarus, 133, 221

Foster, M. J., Green, S. F., McBride, N., \& Davies, J. K. 2000, Icarus, in press

\section{REFERENCES}

Goldreich, P., Murry, N., Longaretti, P. Y., \& Banfield, D. 1989, Science, 245,500

Grundy, W. M., \& Schmitt, B. 1998, J. Geophys. Res., 103, 25809

Hartman, W., Tholen, D., Meech, K., \& Cruikshank, D. 1990, Icarus, 83, 1

Hapke, B. 1981, J. Geophys. Res., 86, 3039

Hardorp, J. 1980, A\&A, 91, 221

Hartmann, W. K. 1987, Icarus, 71, 57

Jewitt, D., \& Kalas, P. 1998, ApJ, 499, L103

Lançon, A., \& Rocca-Volmerange, B. 1992, A\&AS, 96, 593

Levison, H. F., \& Duncan, M. J. 1997, Icarus, 127, 13

Lide, D. R., ed. 1996, CRC Handbook of Chemistry and Physics (Boca Raton, FL: CRC Press)

Luu, J. X., \& Jewitt, D. C. 1990, AJ, 100, 913

Luu, J. X., Jewitt, D. C., \& Cloutis, E. 1994, Icarus, 109, 133

Matthews, K., \& Soifer, B. T. 1994, in Infrared Astronomy with Arrays: The Next Generation, ed. I. McLean (Dordrecht: Kluwer), 239

Owen, T. C., Cruikshank, D. P., Dalle Ore, C. M., Geballe, T. R., Roush, T. L., \& De Bergh, C. 1999, Icarus, 139, 379

Pickering, E. C. 1899, ApJ, 9, 274

Pollack, J. B., Burns, J. A., \& Tauber, M. E. 1979, Icarus, 37, 587

Senay, M. C., \& Jewitt, D. 1994, IAU Circ. 5929

Sykes, M. V., Nelson, B., Cutri, R. M., Kirkpatrick, D. J., Hurt, R., \& Skrutskie, M. 2000, Icarus, submitted

Tholen, D. J., Hartmann, W. K., \& Cruikshank, D. P. 1988, IAU Circ. 4554

Tholen, D. J., \& Zellner, B. 1983, Icarus, 53, 341

. 1984, Icarus, 58, 246

Thomas, P. C., Helfenstein, P., \& Veverka, J. 1991, J. Geophys. Res., 96, 19253

Thomas, P., Veverka, J., Morrison, D., Davies, M., \& Johnson, T. V. 1983, J. Geophys. Res., 88, 8736

van Dishock, E. F., Blake, G. A., Draine, B. T., \& Lunine, J. L. 1993, in Protostars and Planets III, ed. E. H. Levy, J. I. Lunine (Tucson: Univ. Arizona Press), 163

Veverka, J., Brown, R. H. \& Bell, J. F. 1991, in Uranus, ed. J. T. Bergstralh, Miner, E. D., \& Matthews, M. S. (Tucson: Univ. Arizona Press), 528 